magnetic system is attributed to the rearrangement of magnetic microparticles in the chain when applying mechanical pressure to the elastomer. The change of the magnetic flux after exposing the device to a biomechanical stimulus at the level of $100 \mathrm{kPa}$ amounts to $20 \%$, even without the use of any external magnetic field from a permanent or electromagnet (Fig. 1b). The possibility to operate without the magnetic bias is one of the major advantages of the giant magnetoelastic effect, which enables its applicability in portable electronics for energy generation. Indeed, the reversible change of the magnetic flux can be efficiently picked up using an inductive coil, which can transform the time-varying magnetic flux into electromotive force or an electric field. This concept was demonstrated by Zhou and co-workers by coupling the soft magnetic composite to a patterned liquid-metal-based inductive coil (Fig. 1c). This device can transform the biomechanical energy of human motion into electric energy. The resulting magnetoelastic generator is waterproof, mechanically stretchable (Fig. 1c) and can be attached to human skin (Fig. 1d,e) or implanted. Owing to its low impedance of less than $100 \Omega$, the output current density of the harvester is larger than $4 \mathrm{~mA} \mathrm{~cm}^{-2}$, which is sufficient to power wearable and body-implantable devices. The researchers demonstrate that the device can be used to charge capacitors or to power commercial thermometers for continuous monitoring of body temperature (Fig. 1e). Benefiting from the possibility of operating the device in sweaty conditions or even under water, Zhou and colleagues developed a health-monitoring system based on a self-powered pulse wave sensor wirelessly connected to a regular smartphone, which is able to capture all of the cardiovascular parameters to track an individual's health status (Fig. 1f). Owing to the biocompatibility of the rubber matrix, the magnetoelastic generator was also shown to be biocompatible and was validated to work as an implantable power source. To this end, the device was positioned $5 \mathrm{~mm}$ under porcine tissue and mechanically excited using ultrasound radiation at $20 \mathrm{kHz}$, revealing a peak power output of about $30 \mu \mathrm{W}$, which is sufficient to fulfil the power consumption requirements of pacemakers and deep brain stimulators ${ }^{3}$.

It is anticipated that this technology will have a major impact in numerous research and technology fields beyond bioelectronics. For instance, it would be relevant to study the response of this magnetic elastomer in external magnetic fields and quantify its magnetostrictive performance and field-stiffening effects. These properties may be relevant for the use of these materials in untethered, magnetically controlled shape-changing and morphing objects ${ }^{6}$. For these studies, it may be advantageous to reduce the thickness of the composite to enhance its conformability. Along the same lines, combining these magnetic elastomers with soft piezoelectric composites could result in artificial magnetoelectric composites with a large converse magnetoelectric coefficient. Furthermore, these magnetic elastomers with large changes in local magnetic stray fields following mechanical deformation could be included as a component of mechanosensitive electronics for interactive electronic skins, soft actuators and soft robotics. In this respect, it will be useful to combine the soft magnetic composites with soft magnetic field sensors, which will 'translate' the magnetic state of the composite into electrical signals that can be interpreted by flexible electronics ${ }^{7}$. This could become a new interface, enabling haptic feedback following interaction with objects in virtual and augmented reality, as well as a tactile sensor for smart prosthetics and human-machine interfaces ${ }^{8}$. By combining the composite with flexible magnetic switches such as spin valves, artificial magnetoreceptors for interactive energy-efficient electronics can be realized. Finally, another appealing potential application is motion sensing. In this scenario, the soft magnetoelastic generator can operate as a self-powered stretchable strain sensor capturing the motion of fingers, arms or legs for lifestyle, fitness and health applications, such as monitoring Parkinson's disease. The versatility of these simple magnetic composites could be endless.

Denys Makarov (D)

Helmholtz-Zentrum Dresden-Rossendorf e.V.,

Institute of Ion Beam Physics and Materials Research, Dresden, Germany.

$\bigotimes_{e-m a i l: d . m a k a r o v @ h z d r . d e}$

Published online: 23 November 2021 https://doi.org/10.1038/s41563-021-01104-1

References

1. Wang, B., Kostarelos, K., Nelson, B. J. \& Zhang, L. Adv. Mater. 33, 2002047 (2021).

2. Xu, S., Jayaraman, A. \& Rogers, J. A. Nature 571, 319-321 (2019)

3. Yang, S.-Y. et al. Adv. Funct. Mater. https://doi.org/10.1002/adfm. 202009289 (2021).

4. Xu, C., Song, Y., Han, M. \& Zhang, H. Microsyst. Nanoeng. 7, 25 (2021).

5. Zhou, Y. et al. Nat. Mater. https://doi.org/10.1038/s41563-02101093-1 (2021).

6. Wu, S., Hu, W., Ze, Q., Sitti, M. \& Zhao, R. Multifunct. Mater. 3, 042003 (2020).

7. Ha, M. et al. Adv. Mater. 33, 2008751 (2021).

8. Cañón Bermúdez, G. S. \& Makarov, D. Adv. Funct. Mater. https://doi.org/10.1002/adfm.202007788 (2021).

Competing interests

The author declares no competing interests.

\title{
Popping, locking robots
}

\begin{abstract}
An interplay between deswelling and buckling in a polymer gel is harnessed to achieve sequential snap events for repeatable jumping motion, opening the door to autonomously moving soft robots.
\end{abstract}

\section{Johannes T. B. Overvelde}

W e are on the verge of an engineering paradigm shift where we will find ourselves no longer fully understanding - and thus exercising complete control over - the behaviour of the systems that we are developing. We see this transition not only in the field of electrical engineering and computer science, through concepts such as artificial intelligence, but also in materials science where the combination between chemistry and mechanics enables so-called embodied intelligence. The development of responsive materials that can interact with and harvest their environment is an essential step towards 
the development of devices and robots with autonomous functionality. Yet, we have not reached the point where we can truly talk about autonomous behaviour, rather, we still need to find innovative ways to implement actuation, sensing and feedback in such materials to further our understanding. Now, writing in Nature Materials, Kim and colleagues introduce polymer gel discs that harness a mechanical buckling instability to transform a slow deswelling process into a sudden and repeating snapping and jumping motion $^{1}$. It is an elegant experiment that demonstrates how material chemistry and geometry can interact to enable useful and embodied behaviour.

Traditionally, the materials we use in engineering are selected because of their static response. This is in strong contrast to biological systems, for which the ability to respond, adapt and even predict repeatedly has proven to be essential. Such 'intelligent' behaviour can, to some extent, already be seen in plants, which can, for example, orient to follow the motion of the Sun, and even catch insects. For instance, the Venus flytrap is capable of slowly storing elastic energy in its leaves, which can trigger a snapping motion to catch its prey. Here, the use of a mechanical instability is essential, since the osmotic and hydraulic forces that govern the internal processes of plants limit the rate of water transport and with that the speed of water-driven movements ${ }^{2}$. However, embedding such autonomous behaviour in engineered systems has proven to be a challenge. Up until now, researchers have worked on trying to understand the underlying behaviour of these snapping transitions. In fact, they have even been able to harness them in applications such as soft robots ${ }^{3}$, which are robots fabricated from materials with a stiffness resembling human tissue ${ }^{4}$. Yet, in most cases, developed systems still require external energy input and interaction with the environment to trigger the instability ${ }^{5,6}$ and 'reset' the shape to repeat the behaviour. Similar to setting a mouse trap.

Kim and colleagues tackle these challenges by initially considering an experiment on a simple strip of silicone rubber, fully swollen with a solvent. Fuelled through this initial swelling, the solvent in the strip slowly evaporates. This process results in an internal stress that causes the strip to buckle and deform. In turn, this deformation affects the surface area and the corresponding deswelling rate at certain locations, which starts a dance of 'popping' and 'locking' in which continuous deformation and sudden buckling transitions appear after each other (Fig. 1).

To harness the intricate balance between elasticity and evaporation, Kim and
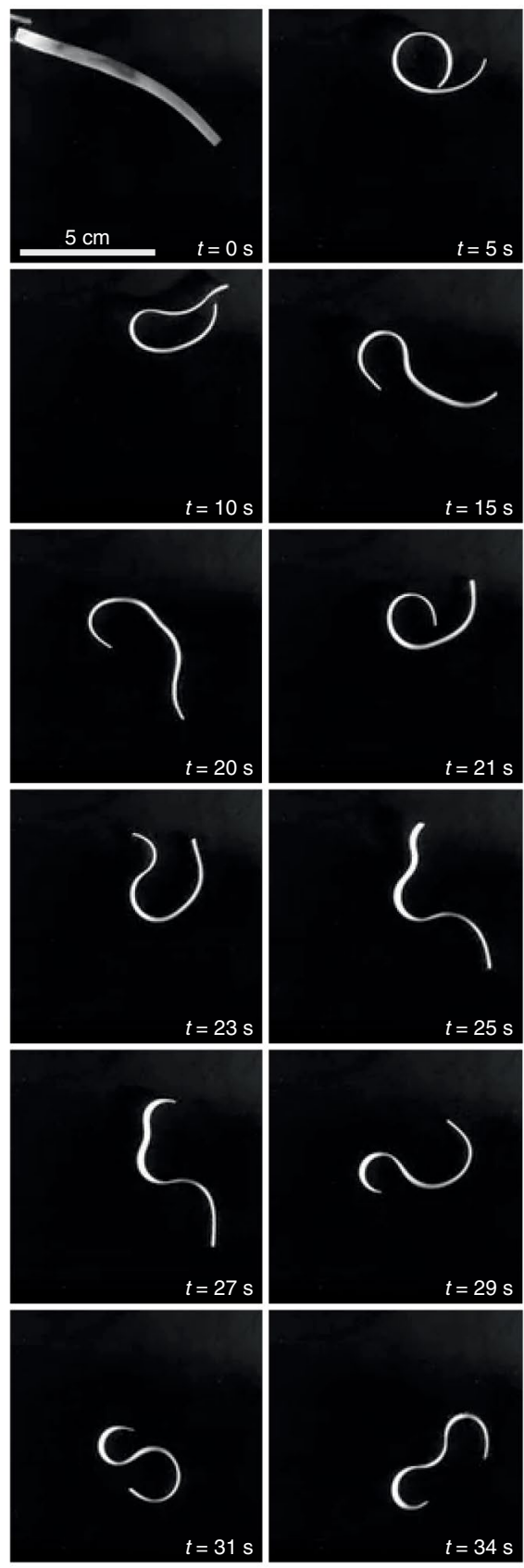

Fig. 1 | Deswelling of a strip of silicone rubber. Snapshots of part of the deswelling process of a strip of silicone rubber initially swollen with a solvent, where continuous deformation and sudden popping events appear after each other. Figure adapted with permission from ref. ${ }^{1}$, Springer Nature Ltd.

co-workers continue their study by casting domes that exhibit a single well-defined motion. When the swollen domes buckle upon evaporation of the solvent, they focus their energy release and jump away from the surface they were sitting on. Importantly, the main feature in these domes now comes from the fact that the domes autonomously 'reset' after each jump, in order to allow for up to 20 pops in a row.

Although we cannot refer to this jumping dome as a fully autonomous soft robot, in particular as there is no direct sensing and feedback with the environment, these experiments illustrate the importance of hysteresis in the response to embody intelligence and achieve potentially useful behaviour. In addition, more complex behaviour could potentially be embedded by considering mechanical interactions between multiple domes, or by embedding fluidic circuits inside the domes that redistribute the solvent ${ }^{7,8}$. Here, a potential way to incorporate sensing might be achieved by harnessing external variations to trigger the instability ${ }^{9}$, in a similar manner to how the Venus flytrap catches an insect. Still, before we will be able to employ this behaviour towards any practical application, some important limitations need to be solved. In particular, the limited number of cycles should be improved by, for example, increasing the efficiency through control of the local evaporation of the solvent. Currently, only $0.0012 \%$ of the evaporated energy is harnessed in the work cycle and thus there is plenty of room for improvement.

Nonetheless, the work by Kim and colleagues provides a foundation towards the development of more autonomous materials and robots. And through their effort, we find ourselves one important step closer to a future where the boundary between living and non-living materials starts to fade. This popping and locking robot opens up the dance floor to all.

\section{Johannes T. B. Overvelde (D) 1,2四 \\ ${ }^{1}$ Autonomous Matter Department, AMOLF, \\ Amsterdam, the Netherlands. ${ }^{2}$ Institute for Complex Molecular Systems and Department of Mechanical Engineering, Eindhoven University of Technology, Eindhoven, the Netherlands.

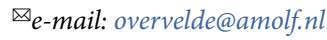

Published online: 23 November 2021 https://doi.org/10.1038/s41563-021-01157-2

References

1. Kim, Y., van den Berg, J. \& Crosby, A. J. Nat. Mater. https://doi. org/10.1038/s41563-020-00909-w (2021).

2. Forterre, Y. J. Exp. Botany 64, 4745-4760 (2013).

3. Gorissen, B., Melancon, D., Vasios, N., Torbati, M. \& Bertoldi, K. Sci. Robot. 20, eabb1967 (2020).

4. Wehner, M. et al. Nature 536, 451-455 (2016).

5. Gelebart, A. H. et al. Nature 546, 632-636 (2017).

6. Hua, M. et al. Matter 4, 1029-1041 (2021).

7. Drotman, D., Jadhav, S., Sharp, D., Chan, C. \& Tolley, M. T. Sci. Robot. 6, eaay2627 (2021)

8. Aubin, C. A. et al. Nature 571, 51-57 (2019)

9. Overvelde, J. T. B., Kloek, T., D’Haen, J. J. A. \& Bertoldi, K. Proc, Natl Acad. Sci. USA 112, 10863-10868 (2015). 